\title{
CONSTRUCTING BISPECTRAL ORTHOGONAL POLYNOMIALS FROM THE CLASSICAL DISCRETE FAMILIES OF CHARLIER, MEIXNER AND KRAWTCHOUK $^{1}$
}

Manuel Domínguez de la Iglesia

Instituto de Matemáticas, C.U.

Universidad Nacional Autónoma de México

Constructive Functions 2014

Vanderbilt University, Nashville, May 26, 2014 


\section{OutLine}

(1) Introduction

- Classical discrete orthogonal polynomials

- Krall orthogonal polynomials

(2) Methodology

- D-operators

- Choice of polynomials

- Identifying the measure

(3) ExAmples

- Charlier, Meixner and Krawtchouk polynomials 


\section{OUTLINE}

(1) Introduction

- Classical discrete orthogonal polynomials

- Krall orthogonal polynomials

- D-operators

- Choice of polynomials

- Identifying the measure

- Charlier, Meixner and Krawtchouk polynomials 


\section{DISCRETE ORTHOGONAL POLYNOMIALS}

A system of polynomials $\left(p_{n}\right)_{n}$ is orthogonal with respect to a discrete measure $\omega(x)=\sum_{x \in \mathcal{S}} a_{x} \delta_{t_{x}}, \mathcal{S} \subset \mathbb{N}$ if

$$
\left\langle p_{n}, p_{m}\right\rangle_{\omega}=\sum_{x \in \mathcal{S}} a_{x} p_{n}\left(t_{x}\right) p_{m}\left(t_{x}\right)=\left\|p_{n}\right\|_{\omega}^{2} \delta_{n m}, \quad n, m \geq 0
$$

Every family of OP's $\left(p_{n}\right)_{n}$ satisfy a three-term recurrence relation

where $a_{n}, c_{n} \neq 0, b_{n} \in \mathbb{R}$ and $p_{0}(x)=1, p_{-1}(x)=0$.

Jacobi operator (tridiagonal):

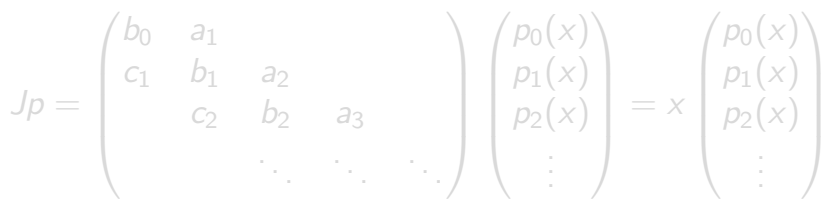

The converse result is also true (Favard's or speçtra! the 


\section{DISCRETE ORTHOGONAL POLYNOMIALS}

A system of polynomials $\left(p_{n}\right)_{n}$ is orthogonal with respect to a discrete measure $\omega(x)=\sum_{x \in \mathcal{S}} a_{x} \delta_{t_{x}}, \mathcal{S} \subset \mathbb{N}$ if

$$
\left\langle p_{n}, p_{m}\right\rangle_{\omega}=\sum_{x \in \mathcal{S}} a_{x} p_{n}\left(t_{x}\right) p_{m}\left(t_{x}\right)=\left\|p_{n}\right\|_{\omega}^{2} \delta_{n m}, \quad n, m \geq 0
$$

Every family of OP's $\left(p_{n}\right)_{n}$ satisfy a three-term recurrence relation

$$
x p_{n}(x)=a_{n+1} p_{n+1}(x)+b_{n} p_{n}(x)+c_{n} p_{n-1}(x), \quad n \geq 1
$$

where $a_{n}, c_{n} \neq 0, b_{n} \in \mathbb{R}$ and $p_{0}(x)=1, p_{-1}(x)=0$.

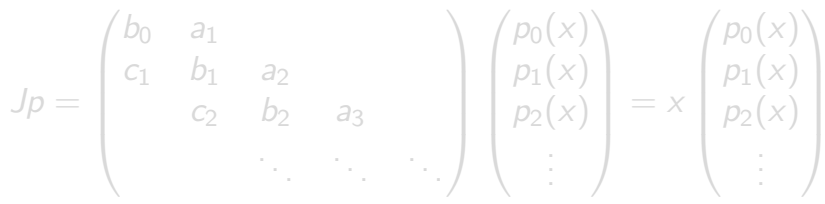




\section{DISCRETE ORTHOGONAL POLYNOMIALS}

A system of polynomials $\left(p_{n}\right)_{n}$ is orthogonal with respect to a discrete measure $\omega(x)=\sum_{x \in \mathcal{S}} a_{x} \delta_{t_{x}}, \mathcal{S} \subset \mathbb{N}$ if

$$
\left\langle p_{n}, p_{m}\right\rangle_{\omega}=\sum_{x \in \mathcal{S}} a_{x} p_{n}\left(t_{x}\right) p_{m}\left(t_{x}\right)=\left\|p_{n}\right\|_{\omega}^{2} \delta_{n m}, \quad n, m \geq 0
$$

Every family of OP's $\left(p_{n}\right)_{n}$ satisfy a three-term recurrence relation

$$
x p_{n}(x)=a_{n+1} p_{n+1}(x)+b_{n} p_{n}(x)+c_{n} p_{n-1}(x), \quad n \geq 1
$$

where $a_{n}, c_{n} \neq 0, b_{n} \in \mathbb{R}$ and $p_{0}(x)=1, p_{-1}(x)=0$. Jacobi operator (tridiagonal):

$$
J p=\left(\begin{array}{ccccc}
b_{0} & a_{1} & & & \\
c_{1} & b_{1} & a_{2} & & \\
& c_{2} & b_{2} & a_{3} & \\
& & \ddots & \ddots & \ddots
\end{array}\right)\left(\begin{array}{c}
p_{0}(x) \\
p_{1}(x) \\
p_{2}(x) \\
\vdots
\end{array}\right)=x\left(\begin{array}{c}
p_{0}(x) \\
p_{1}(x) \\
p_{2}(x) \\
\vdots
\end{array}\right)=x p, \quad x \in \mathcal{S}
$$

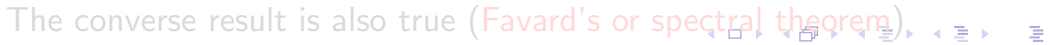




\section{DISCRETE ORTHOGONAL POLYNOMIALS}

A system of polynomials $\left(p_{n}\right)_{n}$ is orthogonal with respect to a discrete measure $\omega(x)=\sum_{x \in \mathcal{S}} a_{x} \delta_{t_{x}}, \mathcal{S} \subset \mathbb{N}$ if

$$
\left\langle p_{n}, p_{m}\right\rangle_{\omega}=\sum_{x \in \mathcal{S}} a_{x} p_{n}\left(t_{x}\right) p_{m}\left(t_{x}\right)=\left\|p_{n}\right\|_{\omega}^{2} \delta_{n m}, \quad n, m \geq 0
$$

Every family of OP's $\left(p_{n}\right)_{n}$ satisfy a three-term recurrence relation

$$
x p_{n}(x)=a_{n+1} p_{n+1}(x)+b_{n} p_{n}(x)+c_{n} p_{n-1}(x), \quad n \geq 1
$$

where $a_{n}, c_{n} \neq 0, b_{n} \in \mathbb{R}$ and $p_{0}(x)=1, p_{-1}(x)=0$. Jacobi operator (tridiagonal):

$$
J p=\left(\begin{array}{ccccc}
b_{0} & a_{1} & & & \\
c_{1} & b_{1} & a_{2} & & \\
& c_{2} & b_{2} & a_{3} & \\
& & \ddots & \ddots & \ddots
\end{array}\right)\left(\begin{array}{c}
p_{0}(x) \\
p_{1}(x) \\
p_{2}(x) \\
\vdots
\end{array}\right)=x\left(\begin{array}{c}
p_{0}(x) \\
p_{1}(x) \\
p_{2}(x) \\
\vdots
\end{array}\right)=x p, \quad x \in \mathcal{S}
$$

The converse result is also true (Favard's or spectral theorem) 


\section{Classical FAMILIES}

If we set

$$
\Delta f(x)=f(x+1)-f(x), \quad \nabla f(x)=f(x)-f(x-1)
$$

the classification problem is to find discrete OP's $\left(p_{n}\right)_{n}$

\section{LANCASTER, 1941}

$$
\begin{aligned}
& \sigma(x) \Delta \nabla p_{n}(x)+\tau(x) \Delta p_{n}(x)+\lambda_{n} p_{n}(x)=0, \quad x \in \mathcal{S} \subset \mathbb{N} \\
& \operatorname{deg} \sigma \leq 2, \quad \operatorname{deg} \tau=1
\end{aligned}
$$

In other words, if we call the shift operator

$$
\mathfrak{s}_{j} f(x)=f(x+j)
$$

the difference equation reads

$$
\begin{aligned}
& {[\sigma(x)+\tau(x)] \mathfrak{s}_{1} p_{n}(x)-[2 \sigma(x)+\tau(x)] \mathfrak{s}_{0} p_{n}(x)} \\
& \quad+\sigma(x) \mathfrak{s}_{-1} p_{n}(x)+\lambda_{n} p_{n}(x)=0, \quad x \in \mathcal{S} \subset \mathbb{N}
\end{aligned}
$$




\section{Classical families}

- Charlier (Poisson): $\mathcal{S}=\{0,1,2, \ldots\}$.

$$
\omega_{a}(x)=\sum_{x=0}^{\infty} \frac{a^{x}}{x !} \delta_{x}, \quad a>0
$$

$$
a c_{n}^{a}(x+1)-(x+a) c_{n}^{a}(x)+x c_{n}^{a}(x-1)=-n c_{n}^{a}(x)
$$

- Meixner (Pascal, Geometric): $S=\{0,1,2, \ldots\}$.

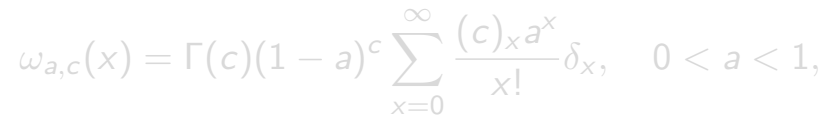

$(i)_{j}=i(i+1) \cdots(i+j-1)$ is the Pochhammer symbol
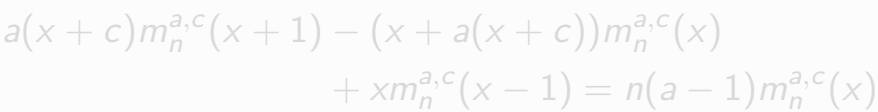


\section{Classical families}

- Charlier (Poisson): $\mathcal{S}=\{0,1,2, \ldots\}$.

$$
\omega_{a}(x)=\sum_{x=0}^{\infty} \frac{a^{x}}{x !} \delta_{x}, \quad a>0
$$

$$
a c_{n}^{a}(x+1)-(x+a) c_{n}^{a}(x)+x c_{n}^{a}(x-1)=-n c_{n}^{a}(x)
$$

- Meixner (Pascal, Geometric): $\mathcal{S}=\{0,1,2, \ldots\}$.

$$
\omega_{a, c}(x)=\Gamma(c)(1-a)^{c} \sum_{x=0}^{\infty} \frac{(c)_{x} a^{x}}{x !} \delta_{x}, \quad 0<a<1, \quad c>0
$$

$(i)_{j}=i(i+1) \cdots(i+j-1)$ is the Pochhammer symbol

$$
\begin{aligned}
a(x+c) m_{n}^{a, c}(x+1) & -(x+a(x+c)) m_{n}^{a, c}(x) \\
& +x m_{n}^{a, c}(x-1)=n(a-1) m_{n}^{a, c}(x)
\end{aligned}
$$




\section{Classical families}

- Krawtchuok (Binomial, Bernoulli): $\mathcal{S}=\{0,1,2, \ldots N-1\}$.

$$
\begin{array}{r}
\omega_{a, N}(x)=\frac{1}{(1+a)^{N-1}} \sum_{x=0}^{N-1}\left(\begin{array}{c}
N-1 \\
x
\end{array}\right) a^{x} \delta_{x}, \quad a>0 \\
a(N-x-1) k_{n}^{a, N}(x+1)-[x+a(N-x-1)] k_{n}^{a, N}(x) \\
+x k_{n}^{a, N}(x-1)=-n(1+a) k_{n}^{a, N}(x)
\end{array}
$$

- Hahn (Hypergeometric): $\mathcal{S}=\{0,1,2, \ldots N\}$

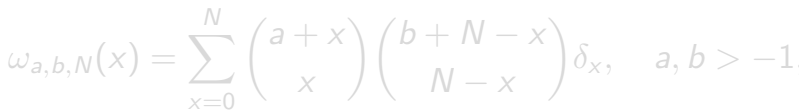




\section{Classical families}

- Krawtchuok (Binomial, Bernoulli): $\mathcal{S}=\{0,1,2, \ldots N-1\}$.

$$
\begin{array}{r}
\omega_{a, N}(x)=\frac{1}{(1+a)^{N-1}} \sum_{x=0}^{N-1}\left(\begin{array}{c}
N-1 \\
x
\end{array}\right) a^{x} \delta_{x}, \quad a>0 \\
a(N-x-1) k_{n}^{a, N}(x+1)-[x+a(N-x-1)] k_{n}^{a, N}(x) \\
+x k_{n}^{a, N}(x-1)=-n(1+a) k_{n}^{a, N}(x)
\end{array}
$$

- Hahn (Hypergeometric): $\mathcal{S}=\{0,1,2, \ldots N\}$

$$
\omega_{a, b, N}(x)=\sum_{x=0}^{N}\left(\begin{array}{c}
a+x \\
x
\end{array}\right)\left(\begin{array}{c}
b+N-x \\
N-x
\end{array}\right) \delta_{x}, \quad a, b>-1, \quad a, b<-N
$$

$$
\begin{aligned}
B(x) h_{n}^{a, b, N}(x+1)- & {[B(x)+D(x)] h_{n}^{a, b, N}(x) } \\
+ & D(x) h_{n}^{a, b, N}(x-1)=n(n+a+b+1) h_{n}^{a, b, N}(x)
\end{aligned}
$$

where $B(x)=(x+a+1)(x-N)$ and $D(x)=x(x-b-N-1)$. 


\section{KRALL POLYNOMIALS (CONTINUOUS CASE)}

GOAL (H.L. Krall, 1939): find families of OP's $\left(q_{n}\right)_{n}$ which are also eigenfunctions of a higher-order differential operator of the form

$$
D_{c}=\sum_{j=0}^{2 m} h_{j}(x) \frac{d^{j}}{d x^{j}}, \quad \operatorname{deg}\left(h_{j}\right) \leq j \quad \Rightarrow \quad D_{c}\left(q_{n}\right)=\lambda_{n} q_{n}
$$

A.M. Krall, Littlejohn, Koornwinder, Koekoek's, Lesky, Grünbaum, Heine, Iliev, Horozov, Zhedanov, etc (80's, 90's, 00's).

$\left(q_{n}\right)_{n}$ are typically orthogonal with respect to the measure 


\section{KRALL POLYNOMIALS (CONTINUOUS CASE)}

GOAL (H.L. Krall, 1939): find families of OP's $\left(q_{n}\right)_{n}$ which are also eigenfunctions of a higher-order differential operator of the form

$$
D_{c}=\sum_{j=0}^{2 m} h_{j}(x) \frac{d^{j}}{d x^{j}}, \quad \operatorname{deg}\left(h_{j}\right) \leq j \quad \Rightarrow \quad D_{c}\left(q_{n}\right)=\lambda_{n} q_{n}
$$

A.M. Krall, Littlejohn, Koornwinder, Koekoek's, Lesky, Grünbaum, Heine, Iliev, Horozov, Zhedanov, etc (80's, 90's, 00's).

$\left(q_{n}\right)_{n}$ are typically orthogonal with respect to the measure 


\section{KRALL POLYNOMials (CONTINUOUS CASE)}

GOAL (H.L. Krall, 1939): find families of OP's $\left(q_{n}\right)_{n}$ which are also eigenfunctions of a higher-order differential operator of the form

$$
D_{c}=\sum_{j=0}^{2 m} h_{j}(x) \frac{d^{j}}{d x^{j}}, \quad \operatorname{deg}\left(h_{j}\right) \leq j \quad \Rightarrow \quad D_{c}\left(q_{n}\right)=\lambda_{n} q_{n}
$$

A.M. Krall, Littlejohn, Koornwinder, Koekoek's, Lesky, Grünbaum, Heine, Iliev, Horozov, Zhedanov, etc (80's, 90's, 00's).

$\left(q_{n}\right)_{n}$ are typically orthogonal with respect to the measure

$$
\omega(x)+\sum_{j=0}^{m-1} a_{j} \delta_{x_{0}}^{(j)}, \quad a_{j} \in \mathbb{R}
$$

where $\omega$ is a (modified) classical weight and $x_{0}$ is an endpoint of the support of orthogonality of $\omega$. 


\section{Krall polynomials (DISCRETE CASE)}

The same question arise in the discrete setting, i.e. find families of OP's $\left(q_{n}\right)_{n}$ which are also eigenfunctions of a higher order difference operator

$$
D_{d}=\sum_{j=r}^{s} h_{j}(x) \mathfrak{s}_{j}, \quad h_{s}, h_{r} \neq 0, \quad \Rightarrow \quad D_{d}\left(q_{n}\right)=\lambda_{n} q_{n}
$$

Bavinck-van Haeringen-Koekoek, 1994: adding deltas at the endpoints of the support does not work (infinite order difference operator).

Surprisingly, it has not been until very recently (Durán, 2012) when the first examples appeared. Also $s-r=2 m$. $\left(q_{n}\right)_{n}$ are typically orthogonal with respect to the measure 


\section{KRALl POLYNOMials (DisCRETE CASE)}

The same question arise in the discrete setting, i.e. find families of OP's $\left(q_{n}\right)_{n}$ which are also eigenfunctions of a higher order difference operator

$$
D_{d}=\sum_{j=r}^{s} h_{j}(x) \mathfrak{s}_{j}, \quad h_{s}, h_{r} \neq 0, \quad \Rightarrow \quad D_{d}\left(q_{n}\right)=\lambda_{n} q_{n}
$$

Bavinck-van Haeringen-Koekoek, 1994: adding deltas at the endpoints of the support does not work (infinite order difference operator).

Surprisingly, it has not been until very recently (Durán, 2012) when the

first examples appeared. Also $s-r=2 m$.

$\left(q_{n}\right)_{n}$ are typically orthogonal with respect to the measure 


\section{Krall polynomials (Discrete CASE)}

The same question arise in the discrete setting, i.e. find families of OP's $\left(q_{n}\right)_{n}$ which are also eigenfunctions of a higher order difference operator

$$
D_{d}=\sum_{j=r}^{s} h_{j}(x) \mathfrak{s}_{j}, \quad h_{s}, h_{r} \neq 0, \quad \Rightarrow \quad D_{d}\left(q_{n}\right)=\lambda_{n} q_{n}
$$

Bavinck-van Haeringen-Koekoek, 1994: adding deltas at the endpoints of the support does not work (infinite order difference operator).

Surprisingly, it has not been until very recently (Durán, 2012) when the first examples appeared. Also $s-r=2 m$.

$\left(q_{n}\right)_{n}$ are typically orthogonal with respect to the measure 


\section{KRALl POLYNOMials (DiscRETE CASE)}

The same question arise in the discrete setting, i.e. find families of OP's $\left(q_{n}\right)_{n}$ which are also eigenfunctions of a higher order difference operator

$$
D_{d}=\sum_{j=r}^{s} h_{j}(x) \mathfrak{s}_{j}, \quad h_{s}, h_{r} \neq 0, \quad \Rightarrow \quad D_{d}\left(q_{n}\right)=\lambda_{n} q_{n}
$$

Bavinck-van Haeringen-Koekoek, 1994: adding deltas at the endpoints of the support does not work (infinite order difference operator).

Surprisingly, it has not been until very recently (Durán, 2012) when the first examples appeared. Also $s-r=2 m$.

$\left(q_{n}\right)_{n}$ are typically orthogonal with respect to the measure

$$
\omega^{F}(x)=\prod_{f \in F}(x-f) \omega(x)
$$

where $\omega$ is a discrete classical weight and $F$ is a finite set of numbers. This is also called a Christoffel transform of $\omega$. 


\section{ConjeCtures (Durán, 2012)}

For a finite set $F$ consider $r_{F}=\sum_{f \in F} f-\frac{n_{F}\left(n_{F}-1\right)}{2}+1$, where $n_{F}=\#(F)$.

Conjecture A: Let wa be the Charlier weight and consider ( $F$ finite)

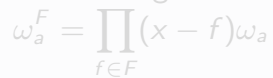

The OP's $\left(q_{n}\right)_{n}$ with respect to $\omega_{a}^{F}$ are eigenfunctions of a higher-order difference operator with $-s=r=r_{F}$.

Conjecture B: Let $\omega_{a, c}$ be the Meixner weight and consider $\left(F_{1}, F_{2}\right.$ finite)

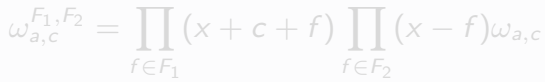

The OP's $\left(q_{n}\right)_{n}$ with respect to $\omega_{a}^{F_{1}, F_{2}}$ are eigenfunctions of a higher-order difference operator with $-s=r=r_{F_{1}}+r_{F_{2}}-1$.

Conjecture $\mathrm{C}$ : Let $\omega_{a, N}$ be the Krawtchouk weight and consider $\left(F_{1}, F_{2}\right.$ finite) $\omega_{a, N}^{F_{1}, F_{2}}=\prod_{f \in F_{1}}(x-f) \prod_{f \in F_{2}}(N-1-f-x) \omega_{a, N}$

The OP's $\left(q_{n}\right)_{n}$ with respect to $w_{a}^{F_{1}, F_{2}}$ are eigenfunctions of a higher-order difference operator with $-s=r=r_{F_{1}}+r_{F_{2}}-1$. 


\section{Conjectures (Durán, 2012)}

For a finite set $F$ consider $r_{F}=\sum_{f \in F} f-\frac{n_{F}\left(n_{F}-1\right)}{2}+1$, where $n_{F}=\#(F)$.

Conjecture A: Let $\omega_{a}$ be the Charlier weight and consider ( $F$ finite)

$$
\omega_{a}^{F}=\prod_{f \in F}(x-f) \omega_{a}
$$

The OP's $\left(q_{n}\right)_{n}$ with respect to $\omega_{a}^{F}$ are eigenfunctions of a higher-order difference operator with $-s=r=r_{F}$.

Conjecture B: Let $\omega_{a, c}$ be the Meixner weight and consider ( $F_{1}, F_{2}$ finite)

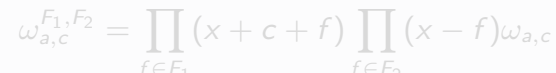

The OP's $\left(q_{n}\right)_{n}$ with respect to $\omega_{a}^{F_{1}, F_{2}}$ are eigenfunctions of a higher-order

difference operator with $-s=r=r_{F_{1}}+r_{F_{2}}-1$.

Conjecture C: Let $\omega_{a, N}$ be the Krawtchouk weight and consider ( $F_{1}, F_{2}$ finite) 


\section{Conjectures (Durán, 2012)}

For a finite set $F$ consider $r_{F}=\sum_{f \in F} f-\frac{n_{F}\left(n_{F}-1\right)}{2}+1$, where $n_{F}=\#(F)$.

Conjecture A: Let $\omega_{a}$ be the Charlier weight and consider ( $F$ finite)

$$
\omega_{a}^{F}=\prod_{f \in F}(x-f) \omega_{a}
$$

The OP's $\left(q_{n}\right)_{n}$ with respect to $\omega_{a}^{F}$ are eigenfunctions of a higher-order difference operator with $-s=r=r_{F}$.

Conjecture B: Let $\omega_{a, c}$ be the Meixner weight and consider $\left(F_{1}, F_{2}\right.$ finite)

$$
\omega_{a, c}^{F_{1}, F_{2}}=\prod_{f \in F_{1}}(x+c+f) \prod_{f \in F_{2}}(x-f) \omega_{a, c}
$$

The OP's $\left(q_{n}\right)_{n}$ with respect to $\omega_{a}^{F_{1}, F_{2}}$ are eigenfunctions of a higher-order difference operator with $-s=r=r_{F_{1}}+r_{F_{2}}-1$. 


\section{Conjectures (Durán, 2012)}

For a finite set $F$ consider $r_{F}=\sum_{f \in F} f-\frac{n_{F}\left(n_{F}-1\right)}{2}+1$, where $n_{F}=\#(F)$.

Conjecture A: Let $\omega_{a}$ be the Charlier weight and consider ( $F$ finite)

$$
\omega_{a}^{F}=\prod_{f \in F}(x-f) \omega_{a}
$$

The OP's $\left(q_{n}\right)_{n}$ with respect to $\omega_{a}^{F}$ are eigenfunctions of a higher-order difference operator with $-s=r=r_{F}$.

Conjecture B: Let $\omega_{a, c}$ be the Meixner weight and consider $\left(F_{1}, F_{2}\right.$ finite)

$$
\omega_{a, c}^{F_{1}, F_{2}}=\prod_{f \in F_{1}}(x+c+f) \prod_{f \in F_{2}}(x-f) \omega_{a, c}
$$

The OP's $\left(q_{n}\right)_{n}$ with respect to $\omega_{a}^{F_{1}, F_{2}}$ are eigenfunctions of a higher-order difference operator with $-s=r=r_{F_{1}}+r_{F_{2}}-1$.

Conjecture C: Let $\omega_{a, N}$ be the Krawtchouk weight and consider $\left(F_{1}, F_{2}\right.$ finite)

$$
\omega_{a, N}^{F_{1}, F_{2}}=\prod_{f \in F_{1}}(x-f) \prod_{f \in F_{2}}(N-1-f-x) \omega_{a, N}
$$

The OP's $\left(q_{n}\right)_{n}$ with respect to $\omega_{a}^{F_{1}, F_{2}}$ are eigenfunctions of a higher-order difference operator with $-s=r=r_{F_{1}}+r_{F_{2}}-1$. 


\section{OUTLINE}

(2) Methodology

- $\mathcal{D}$-operators

- Choice of polynomials

- Identifying the measure

- Charlier, Meixner and Krawtchouk polynomials 


\section{$\mathcal{D}$-OPERATORS}

Let $\mathcal{A}$ be an algebra of (differential or difference) operators and $\left(p_{n}\right)_{n}$ a family of polynomials such that there exists $D_{p} \in \mathcal{A}$ with $D_{p}\left(p_{n}\right)=n p_{n}$. Given a sequence of numbers $\left(\varepsilon_{n}\right)_{n}$, let us consider the operator

$$
\mathcal{D}\left(p_{n}\right)=\sum_{j=1}^{n}(-1)^{j+1} \varepsilon_{n} \cdots \varepsilon_{n-j} p_{n-j}=\varepsilon_{n} p_{n-1}-\varepsilon_{n} \varepsilon_{n-1} p_{n-2}+\cdots
$$

We say that $\mathcal{D}$ is an $\mathcal{D}$-operator associated with $\mathcal{A}$ and $\left(p_{n}\right)_{n}$ if $\mathcal{D} \in \mathcal{A}$. 


\section{$\mathcal{D}$-OPERATORS}

Let $\mathcal{A}$ be an algebra of (differential or difference) operators and $\left(p_{n}\right)_{n}$ a family of polynomials such that there exists $D_{p} \in \mathcal{A}$ with $D_{p}\left(p_{n}\right)=n p_{n}$. Given a sequence of numbers $\left(\varepsilon_{n}\right)_{n}$, let us consider the operator

$$
\mathcal{D}\left(p_{n}\right)=\sum_{j=1}^{n}(-1)^{j+1} \varepsilon_{n} \cdots \varepsilon_{n-j} p_{n-j}=\varepsilon_{n} p_{n-1}-\varepsilon_{n} \varepsilon_{n-1} p_{n-2}+\cdots
$$

We say that $\mathcal{D}$ is an $\mathcal{D}$-operator associated with $\mathcal{A}$ and $\left(p_{n}\right)_{n}$ if $\mathcal{D} \in \mathcal{A}$.

- Laguerre: $\varepsilon_{n}=-1 \Rightarrow \mathcal{D}=\frac{d}{d x}$.
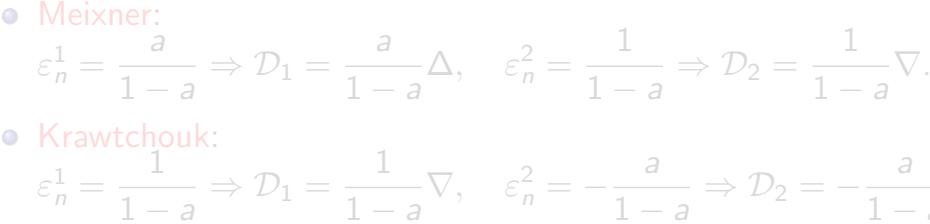


\section{$\mathcal{D}$-OPERATORS}

Let $\mathcal{A}$ be an algebra of (differential or difference) operators and $\left(p_{n}\right)_{n}$ a family of polynomials such that there exists $D_{p} \in \mathcal{A}$ with $D_{p}\left(p_{n}\right)=n p_{n}$. Given a sequence of numbers $\left(\varepsilon_{n}\right)_{n}$, let us consider the operator

$$
\mathcal{D}\left(p_{n}\right)=\sum_{j=1}^{n}(-1)^{j+1} \varepsilon_{n} \cdots \varepsilon_{n-j} p_{n-j}=\varepsilon_{n} p_{n-1}-\varepsilon_{n} \varepsilon_{n-1} p_{n-2}+\cdots
$$

We say that $\mathcal{D}$ is an $\mathcal{D}$-operator associated with $\mathcal{A}$ and $\left(p_{n}\right)_{n}$ if $\mathcal{D} \in \mathcal{A}$.

- Laguerre: $\varepsilon_{n}=-1 \Rightarrow \mathcal{D}=\frac{d}{d x}$.

- Charlier: $\varepsilon_{n}=1 \Rightarrow \mathcal{D}=\nabla$. 


\section{$\mathcal{D}$-OPERATORS}

Let $\mathcal{A}$ be an algebra of (differential or difference) operators and $\left(p_{n}\right)_{n}$ a family of polynomials such that there exists $D_{p} \in \mathcal{A}$ with $D_{p}\left(p_{n}\right)=n p_{n}$. Given a sequence of numbers $\left(\varepsilon_{n}\right)_{n}$, let us consider the operator

$$
\mathcal{D}\left(p_{n}\right)=\sum_{j=1}^{n}(-1)^{j+1} \varepsilon_{n} \cdots \varepsilon_{n-j} p_{n-j}=\varepsilon_{n} p_{n-1}-\varepsilon_{n} \varepsilon_{n-1} p_{n-2}+\cdots
$$

We say that $\mathcal{D}$ is an $\mathcal{D}$-operator associated with $\mathcal{A}$ and $\left(p_{n}\right)_{n}$ if $\mathcal{D} \in \mathcal{A}$.

- Laguerre: $\varepsilon_{n}=-1 \Rightarrow \mathcal{D}=\frac{d}{d x}$.

- Charlier: $\varepsilon_{n}=1 \Rightarrow \mathcal{D}=\nabla$.

- Meixner:

$$
\varepsilon_{n}^{1}=\frac{a}{1-a} \Rightarrow \mathcal{D}_{1}=\frac{a}{1-a} \Delta, \quad \varepsilon_{n}^{2}=\frac{1}{1-a} \Rightarrow \mathcal{D}_{2}=\frac{1}{1-a} \nabla .
$$




\section{$\mathcal{D}$-OPERATORS}

Let $\mathcal{A}$ be an algebra of (differential or difference) operators and $\left(p_{n}\right)_{n}$ a family of polynomials such that there exists $D_{p} \in \mathcal{A}$ with $D_{p}\left(p_{n}\right)=n p_{n}$. Given a sequence of numbers $\left(\varepsilon_{n}\right)_{n}$, let us consider the operator

$$
\mathcal{D}\left(p_{n}\right)=\sum_{j=1}^{n}(-1)^{j+1} \varepsilon_{n} \cdots \varepsilon_{n-j} p_{n-j}=\varepsilon_{n} p_{n-1}-\varepsilon_{n} \varepsilon_{n-1} p_{n-2}+\cdots
$$

We say that $\mathcal{D}$ is an $\mathcal{D}$-operator associated with $\mathcal{A}$ and $\left(p_{n}\right)_{n}$ if $\mathcal{D} \in \mathcal{A}$.

- Laguerre: $\varepsilon_{n}=-1 \Rightarrow \mathcal{D}=\frac{d}{d x}$.

- Charlier: $\varepsilon_{n}=1 \Rightarrow \mathcal{D}=\nabla$.

- Meixner:

$$
\varepsilon_{n}^{1}=\frac{a}{1-a} \Rightarrow \mathcal{D}_{1}=\frac{a}{1-a} \Delta, \quad \varepsilon_{n}^{2}=\frac{1}{1-a} \Rightarrow \mathcal{D}_{2}=\frac{1}{1-a} \nabla .
$$

- Krawtchouk:

$$
\varepsilon_{n}^{1}=\frac{1}{1-a} \Rightarrow \mathcal{D}_{1}=\frac{1}{1-a} \nabla, \quad \varepsilon_{n}^{2}=-\frac{a}{1-a} \Rightarrow \mathcal{D}_{2}=-\frac{a}{1-a} \Delta .
$$




\section{$\mathcal{D}$-OPERATORS}

\section{THEOREM (DURÁN, 2013)}

Let $\mathcal{A},\left(p_{n}\right)_{n}, D_{p}\left(p_{n}\right)=n p_{n},\left(\varepsilon_{n}\right)_{n}$ and $\mathcal{D}$.

For an arbitrary polynomial $R$ such that $R(n) \neq 0, n \geq 0$, we define a new polynomial $P$ by

$$
P(x)-P(x-1)=R(x)
$$

and a sequence of polynomials $\left(q_{n}\right)_{n}$ by $q_{0}=1$ and

$$
q_{n}=p_{n}+\beta_{n} p_{n-1}, \quad n \geq 1
$$

where the numbers $\beta_{n}, n \geq 0$, are given by

$$
\beta_{n}=\varepsilon_{n} \frac{R(n)}{R(n-1)}, \quad n \geq 1
$$

Then there exist $D_{q} \in \mathcal{A}$ sucht that $D_{q}\left(q_{n}\right)=P(n) q_{n}$ where

$$
D_{q}=P\left(D_{p}\right)+\mathcal{D} R\left(D_{p}\right)
$$




\section{$\mathcal{D}$-OPERATORS}

GOAL: Extend the previous Theorem for the case that we consider a linear combination of $m+1$ consecutive $p_{n}$ 's:

$$
q_{n}=p_{n}+\beta_{n, 1} p_{n-1}+\beta_{n, 2} p_{n-2}+\cdots+\beta_{n, m} p_{n-m}
$$

\section{Let $R_{1}, R_{2}, \ldots, R_{m}$ be $m$ arbitrary polynomials and $m$ D-operators $\mathcal{D}_{1}, \mathcal{D}_{2}, \ldots, \mathcal{D}_{m}$ defined by the sequences $\left(\varepsilon_{n}^{h}\right)_{n}, h=1, \ldots, m$.}

\section{Define the auxiliary functions $\xi_{n, i}^{h}$ by}

and assume that the following Casorati determinant never vanish $(n \geq 0)$

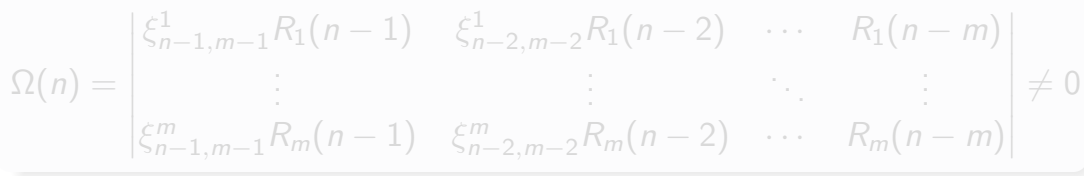




\section{$\mathcal{D}$-OPERATORS}

GOAL: Extend the previous Theorem for the case that we consider a linear combination of $m+1$ consecutive $p_{n}$ 's:

$$
q_{n}=p_{n}+\beta_{n, 1} p_{n-1}+\beta_{n, 2} p_{n-2}+\cdots+\beta_{n, m} p_{n-m}
$$

Let $R_{1}, R_{2}, \ldots, R_{m}$ be $m$ arbitrary polynomials and $m \mathcal{D}$-operators $\mathcal{D}_{1}, \mathcal{D}_{2}, \ldots, \mathcal{D}_{m}$ defined by the sequences $\left(\varepsilon_{n}^{h}\right)_{n}, h=1, \ldots, m$.

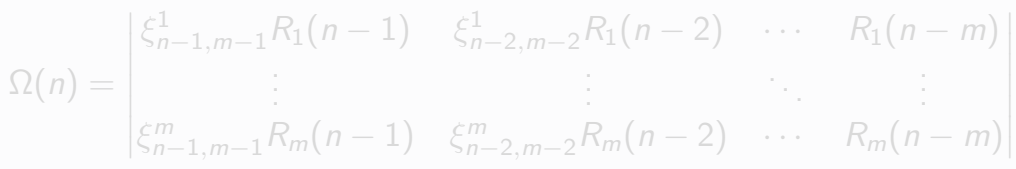




\section{$\mathcal{D}$-OPERATORS}

GOAL: Extend the previous Theorem for the case that we consider a linear combination of $m+1$ consecutive $p_{n}$ 's:

$$
q_{n}=p_{n}+\beta_{n, 1} p_{n-1}+\beta_{n, 2} p_{n-2}+\cdots+\beta_{n, m} p_{n-m}
$$

Let $R_{1}, R_{2}, \ldots, R_{m}$ be $m$ arbitrary polynomials and $m \mathcal{D}$-operators $\mathcal{D}_{1}, \mathcal{D}_{2}, \ldots, \mathcal{D}_{m}$ defined by the sequences $\left(\varepsilon_{n}^{h}\right)_{n}, h=1, \ldots, m$.

Define the auxiliary functions $\xi_{n, i}^{h}$ by

$$
\xi_{n, i}^{h}=\varepsilon_{n}^{h} \varepsilon_{n-1}^{h} \cdots \varepsilon_{n-i+1}^{h}
$$

and assume that the following Casorati determinant never vanish $(n \geq 0)$

$$
\Omega(n)=\left|\begin{array}{cccc}
\xi_{n-1, m-1}^{1} R_{1}(n-1) & \xi_{n-2, m-2}^{1} R_{1}(n-2) & \cdots & R_{1}(n-m) \\
\vdots & \vdots & \ddots & \vdots \\
\xi_{n-1, m-1}^{m} R_{m}(n-1) & \xi_{n-2, m-2}^{m} R_{m}(n-2) & \cdots & R_{m}(n-m)
\end{array}\right| \neq 0
$$




\section{$\mathcal{D}$-OPERATORS}

Now consider the sequence of polynomials $\left(q_{n}\right)_{n}$ defined by

$$
q_{n}(x)=\left|\begin{array}{cccc}
p_{n}(x) & -p_{n-1}(x) & \cdots & (-1)^{m} p_{n-m}(x) \\
\xi_{n, m}^{1} R_{1}(n) & \xi_{n-1, m-1}^{1} R_{1}(n-1) & \cdots & R_{1}(n-m) \\
\vdots & \vdots & \ddots & \vdots \\
\xi_{n, m}^{m} R_{m}(n) & \xi_{n-1, m-1}^{m} R_{m}(n-1) & \cdots & R_{m}(n-m)
\end{array}\right|
$$

Observation: $q_{n}$ is a linear combination of of $m+1$ consecutive $p_{n}$ 's.

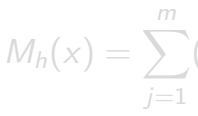




\section{$\mathcal{D}$-OPERATORS}

Now consider the sequence of polynomials $\left(q_{n}\right)_{n}$ defined by

$$
q_{n}(x)=\left|\begin{array}{cccc}
p_{n}(x) & -p_{n-1}(x) & \cdots & (-1)^{m} p_{n-m}(x) \\
\xi_{n, m}^{1} R_{1}(n) & \xi_{n-1, m-1}^{1} R_{1}(n-1) & \cdots & R_{1}(n-m) \\
\vdots & \vdots & \ddots & \vdots \\
\xi_{n, m}^{m} R_{m}(n) & \xi_{n-1, m-1}^{m} R_{m}(n-1) & \cdots & R_{m}(n-m)
\end{array}\right|
$$

Observation: $q_{n}$ is a linear combination of of $m+1$ consecutive $p_{n}$ 's. Define for $h=1, \ldots, m$, the following functions

$$
M_{h}(x)=\sum_{j=1}^{m}(-1)^{h+j} \xi_{x, m-j}^{h} \operatorname{det}\left(\xi_{x+j-r, m-r}^{l} R_{l}(x+j-r)\right)\left\{\begin{array}{l}
l \neq h \\
r \neq j
\end{array}\right\}
$$

Observation: $M_{h}$ are linear combinations of adjoint determinants of $\Omega(x)$.

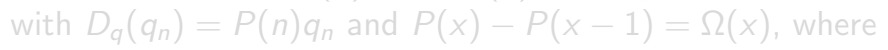




\section{$\mathcal{D}$-OPERATORS}

Now consider the sequence of polynomials $\left(q_{n}\right)_{n}$ defined by

$$
q_{n}(x)=\left|\begin{array}{cccc}
p_{n}(x) & -p_{n-1}(x) & \cdots & (-1)^{m} p_{n-m}(x) \\
\xi_{n, m}^{1} R_{1}(n) & \xi_{n-1, m-1}^{1} R_{1}(n-1) & \cdots & R_{1}(n-m) \\
\vdots & \vdots & \ddots & \vdots \\
\xi_{n, m}^{m} R_{m}(n) & \xi_{n-1, m-1}^{m} R_{m}(n-1) & \cdots & R_{m}(n-m)
\end{array}\right|
$$

Observation: $q_{n}$ is a linear combination of of $m+1$ consecutive $p_{n}$ 's. Define for $h=1, \ldots, m$, the following functions

$$
M_{h}(x)=\sum_{j=1}^{m}(-1)^{h+j} \xi_{x, m-j}^{h} \operatorname{det}\left(\xi_{x+j-r, m-r}^{l} R_{l}(x+j-r)\right)\left\{\begin{array}{l}
l \neq h \\
r \neq j
\end{array}\right\}
$$

Observation: $M_{h}$ are linear combinations of adjoint determinants of $\Omega(x)$. If we assume that $\Omega(x)$ and $M_{h}(x)$ are polynomials in $x$, then $\exists D_{q} \in \mathcal{A}$ with $D_{q}\left(q_{n}\right)=P(n) q_{n}$ and $P(x)-P(x-1)=\Omega(x)$, where

$$
D_{q}=P\left(D_{p}\right)+\sum_{h=1}^{m} M_{h}\left(D_{p}\right) \mathcal{D}_{h} R_{h}\left(D_{p}\right)
$$




\section{Choice of $R_{1}, R_{2}, \ldots, R_{m}$}

GOAL: Make $\left(q_{n}\right)_{n}$ bispectral (we already have $D_{q}\left(q_{n}\right)=\lambda_{n} q_{n}$ ).

For that we have to make an appropriate choice of the arbitrary polynomials $R_{1}, R_{2}, \ldots, R_{m}$. This choice is based on the following recurrence formula $(h=1, \ldots, m)$ :

$\varepsilon_{n+1}^{h} a_{n+1} R_{j}^{h}(n+1)-b_{n} R_{j}^{h}(n)+\frac{c_{n}}{\varepsilon_{n}^{h}} R_{j}^{h}(n-1)=\left(\eta_{h} j+\kappa_{h}\right) R_{j}^{h}(n)$,

where $\eta_{h}$ and $\kappa_{h}$ are real numbers independent of $n$ and $j,\left(a_{n}\right)_{n \in \mathbb{Z}}$, $\left(b_{n}\right)_{n \in \mathbb{Z},}\left(c_{n}\right)_{n \in \mathbb{Z}}$ are the coefficients in the TTRR for the OP's $\left(p_{n}\right)_{n}$. and $\left(\varepsilon_{n}^{h}\right)_{n}$ defines a $\mathcal{D}$-operator for $\left(p_{n}\right)_{n}$.

\begin{tabular}{||l|c|c||}
\hline Classical discrete family & D-operators & $R_{j}(x)$ \\
\hline Charlier: $c_{n}^{a}, n \geq 0$ & $\nabla$ & $c_{j}^{-a}(-x-1), j \geq 0$ \\
\hline Meixner: $m_{n}^{a, c}, n \geq 0$ & $\frac{a}{1-a} \Delta$ & $m_{j}^{1 / a, 2-c}(-x-1), j \geq 0$ \\
\hline & $\frac{1}{1-a} \nabla$ & $m_{j}^{a, 2-c}(-x-1), j \geq 0$ \\
\hline Krawtchouk: $k_{n}^{a, N}, n \geq 0$ & $\frac{1}{1+a} \nabla$ & $k_{j}^{a,-N}(-x-1), j \geq 0$ \\
\hline & $\frac{-a}{1+a} \Delta$ & $k_{j}^{1 / a,-N}(-x-1), j \geq 0$ \\
\hline
\end{tabular}




\section{Choice of $R_{1}, R_{2}, \ldots, R_{m}$}

GOAL: Make $\left(q_{n}\right)_{n}$ bispectral (we already have $D_{q}\left(q_{n}\right)=\lambda_{n} q_{n}$ ).

For that we have to make an appropriate choice of the arbitrary polynomials $R_{1}, R_{2}, \ldots, R_{m}$. This choice is based on the following recurrence formula $(h=1, \ldots, m)$ :

$$
\varepsilon_{n+1}^{h} a_{n+1} R_{j}^{h}(n+1)-b_{n} R_{j}^{h}(n)+\frac{c_{n}}{\varepsilon_{n}^{h}} R_{j}^{h}(n-1)=\left(\eta_{h} j+\kappa_{h}\right) R_{j}^{h}(n), \quad n \in \mathbb{Z}
$$

where $\eta_{h}$ and $\kappa_{h}$ are real numbers independent of $n$ and $j,\left(a_{n}\right)_{n \in \mathbb{Z}}$, $\left(b_{n}\right)_{n \in \mathbb{Z}},\left(c_{n}\right)_{n \in \mathbb{Z}}$ are the coefficients in the TTRR for the OP's $\left(p_{n}\right)_{n}$, and $\left(\varepsilon_{n}^{h}\right)_{n}$ defines a $\mathcal{D}$-operator for $\left(p_{n}\right)_{n}$.

\begin{tabular}{||l|c|c||}
\hline Classical discrete family & D-operators & $R_{j}(x)$ \\
\hline Charlier: $c_{n}^{a}, n \geq 0$ & $\nabla$ & $c_{j}^{-a}(-x-1), j \geq 0$ \\
\hline Meixner: $m_{n}^{a, c}, n \geq 0$ & $\frac{a}{1-a} \Delta$ & $m_{j}^{1 / a, 2-c}(-x-1), j \geq 0$ \\
\hline & $\frac{1}{1-a} \nabla$ & $m_{j}^{a, 2-c}(-x-1), j \geq 0$ \\
\hline Krawtchouk: $k_{n}^{a, N}, n \geq 0$ & $\frac{1}{1+a} \nabla$ & $k_{j}^{a,-N}(-x-1), j \geq 0$ \\
\hline & $\frac{-a}{1+a} \Delta$ & $k_{j}^{1 / a,-N}(-x-1), j \geq 0$ \\
\hline
\end{tabular}




\section{Choice of $R_{1}, R_{2}, \ldots, R_{m}$}

GOAL: Make $\left(q_{n}\right)_{n}$ bispectral (we already have $D_{q}\left(q_{n}\right)=\lambda_{n} q_{n}$ ).

For that we have to make an appropriate choice of the arbitrary polynomials $R_{1}, R_{2}, \ldots, R_{m}$. This choice is based on the following recurrence formula $(h=1, \ldots, m)$ :

$\varepsilon_{n+1}^{h} a_{n+1} R_{j}^{h}(n+1)-b_{n} R_{j}^{h}(n)+\frac{c_{n}}{\varepsilon_{n}^{h}} R_{j}^{h}(n-1)=\left(\eta_{h} j+\kappa_{h}\right) R_{j}^{h}(n), \quad n \in \mathbb{Z}$

where $\eta_{h}$ and $\kappa_{h}$ are real numbers independent of $n$ and $j,\left(a_{n}\right)_{n \in \mathbb{Z}}$, $\left(b_{n}\right)_{n \in \mathbb{Z}},\left(c_{n}\right)_{n \in \mathbb{Z}}$ are the coefficients in the TTRR for the OP's $\left(p_{n}\right)_{n}$, and $\left(\varepsilon_{n}^{h}\right)_{n}$ defines a $\mathcal{D}$-operator for $\left(p_{n}\right)_{n}$.

\begin{tabular}{||l|c|c||}
\hline Classical discrete family & $\mathcal{D}$-operators & $R_{j}(x)$ \\
\hline Charlier: $c_{n}^{a}, n \geq 0$ & $\nabla$ & $c_{j}^{-a}(-x-1), j \geq 0$ \\
\hline Meixner: $m_{n}^{a, c}, n \geq 0$ & $\frac{a}{1-a} \Delta$ & $m_{j}^{1 / a, 2-c}(-x-1), j \geq 0$ \\
\hline & $\frac{1}{1-a} \nabla$ & $m_{j}^{a, 2-c}(-x-1), j \geq 0$ \\
\hline Krawtchouk: $k_{n}^{a, N}, n \geq 0$ & $\frac{1}{1+a} \nabla$ & $k_{j}^{a,-N}(-x-1), j \geq 0$ \\
\hline & $\frac{-a}{1+a} \Delta$ & $k_{j}^{1 / a,-N}(-x-1), j \geq 0$ \\
\hline
\end{tabular}




\section{IDENTIFYING THE MEASURE}

Given a set $G$ of $m$ positive integers, $G=\left\{g_{1}, \ldots, g_{m}\right\}$ we then define the sequence of polynomials $\left(q_{n}^{G}\right)_{n}$ by

$$
q_{n}^{G}(x)=\left|\begin{array}{cccc}
p_{n}(x) & -p_{n-1}(x) & \cdots & (-1)^{m} p_{n-m}(x) \\
\xi_{n, m}^{1} R_{g_{1}}^{1}(n) & \xi_{n-1, m-1}^{1} R_{g_{1}}^{1}(n-1) & \cdots & R_{g_{1}}^{1}(n-m) \\
\vdots & \vdots & \ddots & \vdots \\
\xi_{n, m}^{m} R_{g_{m}}^{m}(n) & \xi_{n-1, m-1}^{m} R_{g_{m}}^{m}(n-1) & \cdots & R_{g_{m}}^{m}(n-m)
\end{array}\right|
$$

$\left(q_{n}^{G}\right)_{n}$ will be orthogonal w.r.t a Christoffel transform of $\omega$ (or several)

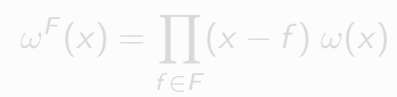

\section{How is the set $G$ related with the set F?: $G$ will be identified by one of}

\section{the following sets:}




\section{IDENTIFYING THE MEASURE}

Given a set $G$ of $m$ positive integers, $G=\left\{g_{1}, \ldots, g_{m}\right\}$ we then define the sequence of polynomials $\left(q_{n}^{G}\right)_{n}$ by

$$
q_{n}^{G}(x)=\left|\begin{array}{cccc}
p_{n}(x) & -p_{n-1}(x) & \cdots & (-1)^{m} p_{n-m}(x) \\
\xi_{n, m}^{1} R_{g_{1}}^{1}(n) & \xi_{n-1, m-1}^{1} R_{g_{1}}^{1}(n-1) & \cdots & R_{g_{1}}^{1}(n-m) \\
\vdots & \vdots & \ddots & \vdots \\
\xi_{n, m}^{m} R_{g_{m}}^{m}(n) & \xi_{n-1, m-1}^{m} R_{g_{m}}^{m}(n-1) & \cdots & R_{g_{m}}^{m}(n-m)
\end{array}\right|
$$

$\left(q_{n}^{G}\right)_{n}$ will be orthogonal w.r.t a Christoffel transform of $\omega$ (or several)

$$
\omega^{F}(x)=\prod_{f \in F}(x-f) \omega(x)
$$




\section{IDENTIFYING THE MEASURE}

Given a set $G$ of $m$ positive integers, $G=\left\{g_{1}, \ldots, g_{m}\right\}$ we then define the sequence of polynomials $\left(q_{n}^{G}\right)_{n}$ by

$$
q_{n}^{G}(x)=\left|\begin{array}{cccc}
p_{n}(x) & -p_{n-1}(x) & \cdots & (-1)^{m} p_{n-m}(x) \\
\xi_{n, m}^{1} R_{g_{1}}^{1}(n) & \xi_{n-1, m-1}^{1} R_{g_{1}}^{1}(n-1) & \cdots & R_{g_{1}}^{1}(n-m) \\
\vdots & \vdots & \ddots & \vdots \\
\xi_{n, m}^{m} R_{g_{m}}^{m}(n) & \xi_{n-1, m-1}^{m} R_{g_{m}}^{m}(n-1) & \cdots & R_{g_{m}}^{m}(n-m)
\end{array}\right|
$$

$\left(q_{n}^{G}\right)_{n}$ will be orthogonal w.r.t a Christoffel transform of $\omega$ (or several)

$$
\omega^{F}(x)=\prod_{f \in F}(x-f) \omega(x)
$$

How is the set $G$ related with the set $F$ ?: $G$ will be identified by one of the following sets:

$$
\begin{aligned}
I(F) & =\left\{1,2, \ldots, f_{k}\right\} \backslash\left\{f_{k}-f, f \in F\right\}, \\
J_{h}(F) & =\left\{0,1,2, \ldots, f_{k}+h-1\right\} \backslash\{f-1, f \in F\}, \quad h \geq 1
\end{aligned}
$$

where $f_{k}=\max F$ and $k=\#(F)$. 


\section{Outline}

- Classical discrete orthogonal polynomials

- Krall orthogonal polynomials

(2) MethodologY

- D-operators

- Choice of polynomials

- Identifying the measure

(3) ExAmples

- Charlier, Meixner and Krawtchouk polynomials 


\section{CHARLIER POLYNOMIALS}

Let $F \subset \mathbb{N}$ be finite and consider $G=I(F)=\left\{g_{1}, \ldots, g_{m}\right\}$. Let $\omega_{a}$ be the Charlier measure and $\left(c_{m}^{a}\right)_{n}$ its sequence of OP's. Assume that $\Omega_{G}(n)=\operatorname{det}\left(c_{g_{l}}^{-a}(-n-j-1)\right)_{l, j=1}^{m} \neq 0$.

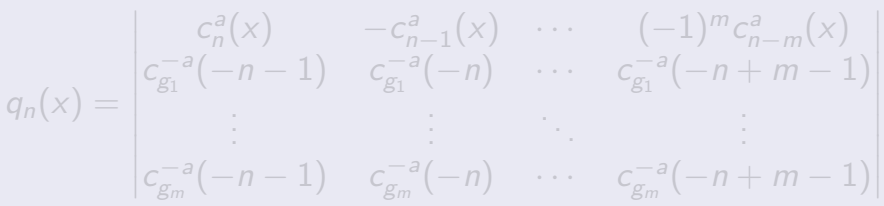

then the polynomials $\left(q_{n}\right)_{n}$ are orthogonal with respect to the measure

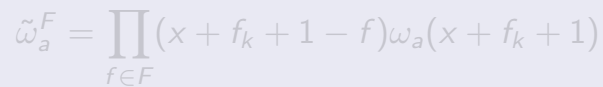

and they are eigenfunctions of a higher order difference operator $D_{q}$ with $-s=r=\sum_{f \in F} f-\frac{n_{F}\left(n_{F}-1\right)}{2}+1$, where $n_{F}=\#(F)$ and $f_{k}=\max F$ Proof of Conjecture $A: \omega_{a}^{F}=a^{f_{k}+1} \tilde{\omega}_{a}^{F}\left(x-f_{k}-1\right)$. 


\section{CHARLIER POLYNOMials}

Let $F \subset \mathbb{N}$ be finite and consider $G=I(F)=\left\{g_{1}, \ldots, g_{m}\right\}$.

Let $\omega_{a}$ be the Charlier measure and $\left(c_{n}^{a}\right)_{n}$ its sequence of OP's. Assume that $\Omega_{G}(n)=\operatorname{det}\left(c_{g_{l}}^{-a}(-n-j-1)\right)_{l, j=1}^{m} \neq 0$.

If we define $\left(q_{n}\right)_{n}$ by

$$
q_{n}(x)=\left|\begin{array}{cccc}
c_{n}^{a}(x) & -c_{n-1}^{a}(x) & \cdots & (-1)^{m} c_{n-m}^{a}(x) \\
c_{g_{1}}^{-a}(-n-1) & c_{g_{1}}^{-a}(-n) & \cdots & c_{g_{1}}^{-a}(-n+m-1) \\
\vdots & \vdots & \ddots & \vdots \\
c_{g_{m}}^{-a}(-n-1) & c_{g_{m}}^{-a}(-n) & \cdots & c_{g_{m}}^{-a}(-n+m-1)
\end{array}\right|
$$

then the polynomials $\left(q_{n}\right)_{n}$ are orthogonal with respect to the measure

$$
\tilde{\omega}_{a}^{F}=\prod_{f \in F}\left(x+f_{k}+1-f\right) \omega_{a}\left(x+f_{k}+1\right)
$$

and they are eigenfunctions of a higher order difference operator $D_{q}$ with $-s=r=\sum_{f \in F} f-\frac{n_{F}\left(n_{F}-1\right)}{2}+1$, where $n_{F}=\#(F)$ and $f_{k}=\max F$. 


\section{CHARLIER POLYNOMials}

Let $F \subset \mathbb{N}$ be finite and consider $G=I(F)=\left\{g_{1}, \ldots, g_{m}\right\}$.

Let $\omega_{a}$ be the Charlier measure and $\left(c_{m}^{a}\right)_{n}$ its sequence of OP's. Assume that $\Omega_{G}(n)=\operatorname{det}\left(c_{g_{l}}^{-a}(-n-j-1)\right)_{l, j=1}^{m} \neq 0$.

If we define $\left(q_{n}\right)_{n}$ by

$$
q_{n}(x)=\left|\begin{array}{cccc}
c_{n}^{a}(x) & -c_{n-1}^{a}(x) & \cdots & (-1)^{m} c_{n-m}^{a}(x) \\
c_{g_{1}}^{-a}(-n-1) & c_{g_{1}}^{-a}(-n) & \cdots & c_{g_{1}}^{-a}(-n+m-1) \\
\vdots & \vdots & \ddots & \vdots \\
c_{g_{m}}^{-a}(-n-1) & c_{g_{m}}^{-a}(-n) & \cdots & c_{g_{m}}^{-a}(-n+m-1)
\end{array}\right|
$$

then the polynomials $\left(q_{n}\right)_{n}$ are orthogonal with respect to the measure

$$
\tilde{\omega}_{a}^{F}=\prod_{f \in F}\left(x+f_{k}+1-f\right) \omega_{a}\left(x+f_{k}+1\right)
$$

and they are eigenfunctions of a higher order difference operator $D_{q}$ with $-s=r=\sum_{f \in F} f-\frac{n_{F}\left(n_{F}-1\right)}{2}+1$, where $n_{F}=\#(F)$ and $f_{k}=\max F$.

Proof of Conjecture A: $\omega_{a}^{F}=a^{f_{k}+1} \tilde{\omega}_{a}^{F}\left(x-f_{k}-1\right)$. 


\section{ChaRlier POLYNOMIALS: EXPLICIT EXAMPLE}

Let $a=1, F=\{1,3\}, G=I(F)=\{1,3\}$.

$\frac{q_{n}^{G}}{\Omega(n)}=c_{n}^{1}+\beta_{n, 1} c_{n-1}^{1}+\beta_{n, 2} c_{n-2}^{1}$ are orthogonal w.r.t

$$
\tilde{\omega}_{1}^{F}=(x+3)(x+1) \omega_{1}(x+4)
$$

The difference operator (of order 8 ) satisfying $D_{q}\left(q_{n}^{G}\right)=P(n) q_{n}^{G}$ is

$$
D_{q}=P\left(D_{1}\right)+M_{1}\left(D_{1}\right) \nabla R_{1}\left(D_{1}\right)+M_{2}\left(D_{1}\right) \nabla R_{2}\left(D_{1}\right)
$$

where

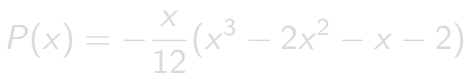




\section{CHARLIER POLYNOMIALS: EXPLICIT EXAMPLE}

Let $a=1, F=\{1,3\}, G=I(F)=\{1,3\}$.

$\frac{q_{n}^{G}}{\Omega(n)}=c_{n}^{1}+\beta_{n, 1} c_{n-1}^{1}+\beta_{n, 2} c_{n-2}^{1}$ are orthogonal w.r.t

$$
\tilde{\omega}_{1}^{F}=(x+3)(x+1) \omega_{1}(x+4)
$$

The difference operator (of order 8) satisfying $D_{q}\left(q_{n}^{G}\right)=P(n) q_{n}^{G}$ is

$$
D_{q}=P\left(D_{1}\right)+M_{1}\left(D_{1}\right) \nabla R_{1}\left(D_{1}\right)+M_{2}\left(D_{1}\right) \nabla R_{2}\left(D_{1}\right)
$$

where

$$
\begin{gathered}
D_{1}=-x \mathfrak{s}_{-1}+(x+1) \mathfrak{s}_{0}-\mathfrak{s}_{1}, \quad D_{1}\left(c_{n}^{1}\right)=n c_{n}^{1}, \quad n \geq 0 \\
R_{1}(x)=-x, \quad R_{2}(x)=-\frac{1}{6}\left(x^{3}+3 x^{2}+5 x+2\right) \\
M_{1}(x)=x^{2}+2 x+2, \quad M_{2}(x)=-2 \\
P(x)=-\frac{x}{12}\left(x^{3}-2 x^{2}-x-2\right)
\end{gathered}
$$




\section{MEIXNER POLYNOMIALS}

In this case have two different $\mathcal{D}$-operators. That means that we will have to consider two sets of positive integers $F_{1}, F_{2} \subset \mathbb{N}$.

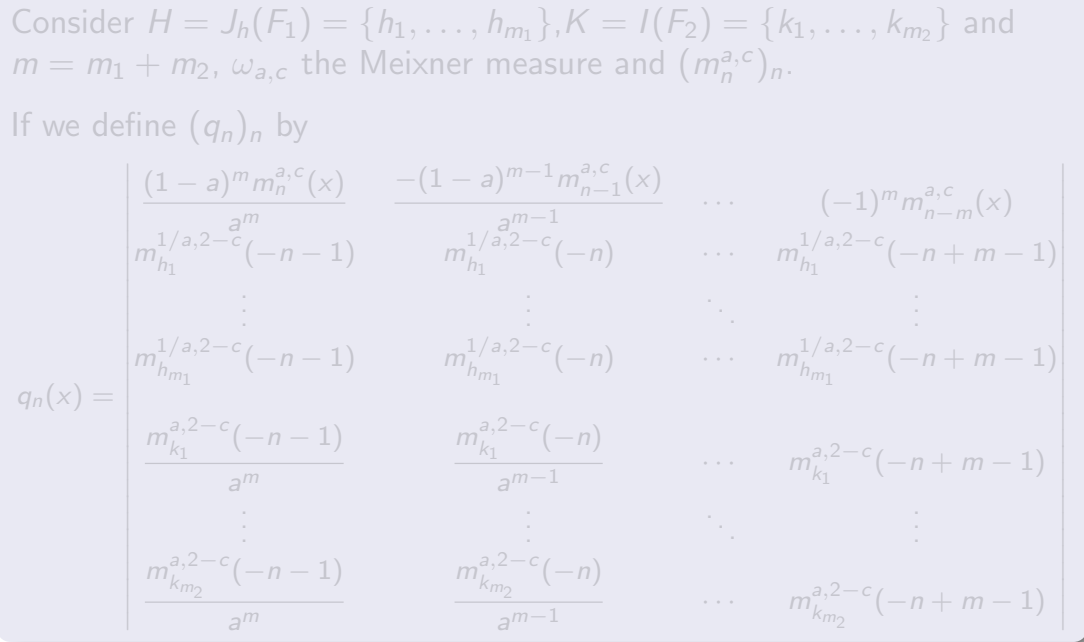




\section{MEIXNER POLYNOMIALS}

In this case have two different $\mathcal{D}$-operators. That means that we will have to consider two sets of positive integers $F_{1}, F_{2} \subset \mathbb{N}$.

Consider $H=J_{h}\left(F_{1}\right)=\left\{h_{1}, \ldots, h_{m_{1}}\right\}, K=I\left(F_{2}\right)=\left\{k_{1}, \ldots, k_{m_{2}}\right\}$ and $m=m_{1}+m_{2}, \omega_{a, c}$ the Meixner measure and $\left(m_{n}^{a, c}\right)_{n}$.

If we define $\left(q_{n}\right)_{n}$ by

$$
q_{n}(x)=\left|\begin{array}{cccc}
\frac{(1-a)^{m} m_{n}^{a, c}(x)}{a^{m}} & \frac{-(1-a)^{m-1} m_{n-1}^{a, c}(x)}{n m_{h_{1}}^{1 / a, 2-c}(-n-1)} & \cdots & (-1)^{m} m_{n-m}^{a, c}(x) \\
\vdots & m_{h_{1}}^{1 / a, 2-c}(-n) & \cdots & m_{h_{1}}^{1 / a, 2-c}(-n+m-1) \\
m_{h_{m_{1}}}^{1 / a, 2-c}(-n-1) & m_{h_{m_{1}}}^{1 / a, 2-c}(-n) & \cdots & m_{h_{m_{1}}}^{1 / a, 2-c}(-n+m-1) \\
\frac{m_{k_{1}}^{a, 2-c}(-n-1)}{a^{m}} & \frac{m_{k_{1}}^{a, 2-c}(-n)}{a^{m-1}} & \cdots & m_{k_{1}}^{a, 2-c}(-n+m-1) \\
\vdots & \frac{m_{k_{m_{2}}}^{a, 2-c}(-n)}{a^{m-1}} & \cdots & \vdots \\
\frac{m_{k_{m_{2}}}^{a, 2-c}(-n-1)}{a^{m}} & \frac{1}{a^{m}} & \cdots & m_{k_{m_{2}}}^{a, 2-c}(-n+m-1)
\end{array}\right|
$$


Then the polynomials $\left(q_{n}\right)_{n}$ are orthogonal with respect to the measure

$$
\tilde{\omega}_{a, c}^{F_{1}, F_{2}, h}=\prod_{f \in F_{1}}(x+c-f) \prod_{f \in F_{2}}\left(x+f_{2, M}+1-f\right) \omega_{a, c-f_{1, M}-f_{2, M}-h-1}\left(x+f_{2, M}+1\right)
$$
and they are eigenfunctions of a higher order difference operator $D_{q}$ with

$$
-s=r=\sum_{f \in F_{2}} f-\sum_{f \in F_{1}} f-\frac{n_{F_{1}}\left(n_{F_{1}}-1\right)}{2}-\frac{n_{F_{2}}\left(n_{F_{2}}-1\right)}{2}+n_{F_{1}}\left(f_{1, M}+h\right)+1
$$

where $n_{F_{i}}=\#\left(F_{i}\right)$ and $f_{i, M}=\max F_{i}, i=1,2$. 


\section{MEIXNER POLYNOMIALS}

Then the polynomials $\left(q_{n}\right)_{n}$ are orthogonal with respect to the measure

$$
\tilde{\omega}_{a, c}^{F_{1}, F_{2}, h}=\prod_{f \in F_{1}}(x+c-f) \prod_{f \in F_{2}}\left(x+f_{2, M}+1-f\right) \omega_{a, c-f_{1, M}-f_{2, M}-h-1}\left(x+f_{2, M}+1\right)
$$
and they are eigenfunctions of a higher order difference operator $D_{q}$ with

$$
-s=r=\sum_{f \in F_{2}} f-\sum_{f \in F_{1}} f-\frac{n_{F_{1}}\left(n_{F_{1}}-1\right)}{2}-\frac{n_{F_{2}}\left(n_{F_{2}}-1\right)}{2}+n_{F_{1}}\left(f_{1, M}+h\right)+1
$$

where $n_{F_{i}}=\#\left(F_{i}\right)$ and $f_{i, M}=\max F_{i}, i=1,2$.

Proof of Conjecture B: Write $\tilde{F}_{1}=\left\{f_{1, M}-f+1, f \in F_{1}\right\}$,

$\tilde{c}=c+f_{1, M}+f_{2, M}+2$ and $h=\min F_{1}$. In particular $J_{h}\left(\tilde{F}_{1}\right)=I\left(F_{1}\right)$.

Therefore we have

$$
\omega_{a, c}^{F_{1}, F_{2}}=(1-a)^{c-\tilde{c}} \tilde{\omega}_{a, \tilde{c}}^{\tilde{F}_{1}, F_{2}, h}\left(x-f_{2, M}-1\right)
$$




\section{KRAWTCHOUK POLYNOMIALS}

Again we have two different $\mathcal{D}$-operators. Consider $F_{1}, F_{2} \subset \mathbb{N}$ finite and $K=I\left(F_{1}\right)=\left\{k_{1}, \ldots, k_{m_{2}}\right\}, H=J_{h}\left(F_{2}\right)=\left\{h_{1}, \ldots, h_{m_{1}}\right\}, m=m_{1}+m_{2}$, $\omega_{a, N}$ the Krawtchouk measure and $\left(k_{n}^{a, N}\right)_{n}$. We assume that $f_{1, M}, f_{2, M}<N / 2$ (so that $F_{1} \cap\left\{N-1-f, f \in F_{2}\right\}=\emptyset$ ), where $f_{i, M}=\max F_{i}$.

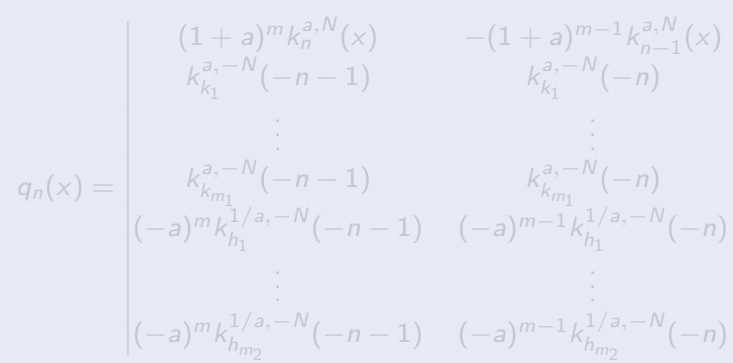




\section{KRAWTCHOUK POLYNOMIALS}

Again we have two different $\mathcal{D}$-operators. Consider $F_{1}, F_{2} \subset \mathbb{N}$ finite and $K=I\left(F_{1}\right)=\left\{k_{1}, \ldots, k_{m_{2}}\right\}, H=J_{h}\left(F_{2}\right)=\left\{h_{1}, \ldots, h_{m_{1}}\right\}, m=m_{1}+m_{2}$, $\omega_{a, N}$ the Krawtchouk measure and $\left(k_{n}^{a, N}\right)_{n}$. We assume that $f_{1, M}, f_{2, M}<N / 2$ (so that $F_{1} \cap\left\{N-1-f, f \in F_{2}\right\}=\emptyset$ ), where $f_{i, M}=\max F_{i}$. If we define $\left(q_{n}\right)_{n}$ by

$$
q_{n}(x)=\left|\begin{array}{cccc}
(1+a)^{m} k_{n}^{a, N}(x) & -(1+a)^{m-1} k_{n-1}^{a, N}(x) & \cdots & (-1)^{m} k_{n-m}^{a, N}(x) \\
k_{k_{1}}^{a,-N}(-n-1) & k_{k_{1}}^{a,-N}(-n) & \cdots & k_{k_{1}}^{a,-N}(-n+m-1) \\
\vdots & \vdots & \ddots & \vdots \\
k_{k_{m_{1}}}^{a,-N}(-n-1) & k_{k_{m_{1}}}^{a,-N}(-n) & \cdots & k_{k_{m_{1}}}^{a,-N}(-n+m-1) \\
(-a)^{m} k_{h_{1}}^{1 / a,-N}(-n-1) & (-a)^{m-1} k_{h_{1}}^{1 / a,-N}(-n) & \cdots & k_{h_{1}}^{1 / a,-N}(-n+m-1) \\
\vdots & \vdots & \ddots & \vdots \\
(-a)^{m} k_{h_{m_{2}}}^{1 / a,-N}(-n-1) & (-a)^{m-1} k_{h_{m_{2}}}^{1 / a,-N}(-n) & \cdots & k_{h_{m_{2}}}^{1 / a,-N}(-n+m-1)
\end{array}\right|
$$




\section{KRAWTCHOUK POLYNOMIALS}

Then the polynomials $\left(q_{n}\right)_{n}$ are orthogonal with respect to the measure

$$
\tilde{\omega}_{a, N}^{F_{1}, F_{2}, h}=\prod_{f \in F_{1}}\left(x+f_{1, M}+1-f\right) \prod_{f \in F_{2}}(N-x-1+f) \omega_{a, N+f_{1, M}+f_{2, M}+h+1}\left(x+f_{1, M}+1\right)
$$

and they are eigenfunctions of a higher order difference operator $D_{q}$ with

$$
-s=r=\sum_{f \in F_{1}} f-\sum_{f \in F_{2}} f-\frac{n_{F_{1}}\left(n_{F_{1}}-1\right)}{2}-\frac{n_{F_{2}}\left(n_{F_{2}}-1\right)}{2}+n_{F_{2}}\left(f_{2, M}+h\right)+1
$$

where $n_{F_{i}}=\#\left(F_{i}\right)$ and $f_{i, M}=\max F_{i}, i=1,2$. 


\section{KRAWTCHOUK POLYNOMIALS}

Then the polynomials $\left(q_{n}\right)_{n}$ are orthogonal with respect to the measure

$$
\tilde{\omega}_{a, N}^{F_{1}, F_{2}, h}=\prod_{f \in F_{1}}\left(x+f_{1, M}+1-f\right) \prod_{f \in F_{2}}(N-x-1+f) \omega_{a, N+f_{1, M}+f_{2, M}+h+1}\left(x+f_{1, M}+1\right)
$$

and they are eigenfunctions of a higher order difference operator $D_{q}$ with

$$
-s=r=\sum_{f \in F_{1}} f-\sum_{f \in F_{2}} f-\frac{n_{F_{1}}\left(n_{F_{1}}-1\right)}{2}-\frac{n_{F_{2}}\left(n_{F_{2}}-1\right)}{2}+n_{F_{2}}\left(f_{2, M}+h\right)+1
$$

where $n_{F_{i}}=\#\left(F_{i}\right)$ and $f_{i, M}=\max F_{i}, i=1,2$.

Proof of Conjecture C: Write $\tilde{F}_{1}=\left\{f_{1, M}-f+1, f \in F_{1}\right\}$, $\tilde{F}_{2}=\left\{f_{1, M}+f_{2, M}-f+2, f \in F_{2}\right\}, \tilde{N}=N-f_{1, M}-f_{2, M}-2$ and $h=\min F_{1}$. In particular $J_{h}\left(\tilde{F}_{2}\right)=I\left(F_{2}\right)$. Therefore we have

$$
\omega_{a, N}^{F_{1}, F_{2}}=\tilde{\omega}_{a, \tilde{N}}^{\tilde{F}_{1}, \tilde{F}_{2}, h}\left(x-f_{1, M}-1\right)
$$

